\title{
Assessment of the non-hydrostatic effect on the upper atmosphere using a general circulation model (GCM)
}

\author{
Yue Deng, ${ }^{1}$ Arthur D. Richmond, ${ }^{1}$ Aaron J. Ridley, ${ }^{2}$ and Han-Li Liu ${ }^{1}$ \\ Received 3 October 2007; revised 27 November 2007; accepted 7 December 2007; published 11 January 2008.
}

[1] Under hydrostatic equilibrium, a typical assumption used in global thermosphere ionosphere models, the pressure gradient in the vertical direction is exactly balanced by the gravity force. Using the non-hydrostatic Global Ionosphere Thermosphere Model (GITM), which solves the complete vertical momentum equation, the primary characteristics of non-hydrostatic effects on the upper atmosphere are investigated. Our results show that after a sudden intense enhancement of high-latitude Joule heating, the vertical pressure gradient force can locally be $25 \%$ larger than the gravity force, resulting in a significant disturbance away from hydrostatic equilibrium. This disturbance is transported from the lower altitude source region to high altitudes through an acoustic wave, which has been simulated in a global circulation model for the first time. Due to the conservation of perturbation energy, the magnitude of the vertical wind perturbation increases with altitude and reaches $150(250) \mathrm{m} / \mathrm{s}$ at 300 (430) $\mathrm{km}$ during the disturbance. The upward neutral wind lifts the atmosphere and raises the neutral density at high altitudes by more than $100 \%$. These large vertical winds are not typically reproduced by hydrostatic models of the thermosphere and ionosphere. Our results give an explanation of the cause of such strong vertical winds reported in many observations. Citation: Deng, Y., A. D. Richmond, A. J. Ridley, and H.-L. Liu (2008), Assessment of the non-hydrostatic effect on the upper atmosphere using a general circulation model (GCM), Geophys. Res. Lett., 35, L01104, doi:10.1029/2007GL032182.

\section{Introduction}

[2] Many theoretical thermosphere/ionosphere models have been developed since 1970s, including Thermospheric General Circulation Model (TGCM) [Dickinson et al., 1981], Coupled Thermosphere Ionosphere Model (CTIM) [Fuller-Rowell and Rees, 1980] and their later variants. One common assumption used in these models is the hydrostatic equilibrium, under which the pressure gradient force is balanced with the gravity force in the vertical direction:

$$
\frac{\partial P}{\partial r}=\rho \mathbf{g}
$$

where $P$ is the pressure, $r$ is the radial distance, $\rho$ is the mass density, and $\mathbf{g}$ is gravitation acceleration. If the hydrostatic

\footnotetext{
${ }^{1}$ High Altitude Observatory, National Center for Atmospheric Research, Boulder, Colorado, USA.

${ }^{2}$ Center for Space Environment Modeling, University of Michigan, Ann Arbor, Michigan, USA.
}

Copyright 2008 by the American Geophysical Union. 0094-8276/08/2007GL032182 approximation is relaxed, the vertical momentum can be expanded to:

$$
\frac{\partial u_{r}}{\partial t}+\mathbf{u} \cdot \nabla u_{r}+\frac{1}{\rho} \frac{\partial P}{\partial r}=\mathbf{g}+F_{f}+F_{c},
$$

where $u_{r}$ is the vertical component of the neutral wind, $\mathbf{u}$ is neutral wind vector, $F_{f}$ contains the forces due to ion-neutral and neutral-neutral friction (when each constituent is solved independently), and $F_{c}$ contains centrifugal and Coriolis forces. Compared with equation 1, equation 2 has more terms to bring the non-hydrostatic effects in the system, which can propagate vertically. In order to truly understand both Earth and other planetary atmospheres, the hydrodynamic phenomena should be investigated. Recently, some studies have been done in the low atmosphere (troposphere) using non-hydrostatic models, such as WRF [Skamarock and Klemp, 2007], but very little attention has been paid to the non-hydrostatic effect on the upper atmosphere. Using the newly developed Global Ionosphere Thermosphere Model (GITM) [Ridley et al., 2006], the non-hydrostatic effect on the upper atmosphere has been investigated and quantified in this study, and the acoustic wave has been simulated in a global circulation model for the first time.

[3] Many papers have reported strong vertical winds (more than $100 \mathrm{~m} / \mathrm{s}$ ) in thermosphere from Dynamics Explorer 2 (DE 2) satellite [Innis and Conde, 2002] and Fabry-Perot interferometers (FPI) measurements [Smith and Hernandez, 1995; Aruliah et al., 2005]. Such large vertical winds cause strong disturbance of neutral density in the upper atmosphere, which can dramatically alter low-altitude satellite orbits through increasing the atmospheric drag on the satellites. The proposed drivers for the large vertical wind include localized heating [Price et al., 1995], divergence in the horizontal wind [Smith and Hernandez, 1995] and acoustic-gravity waves [Innis and Conde, 2002]. However, the vertical winds in hydrostatic general circulation models (GCMs), calculated from the divergence of the horizontal wind field, are usually less than $20 \mathrm{~m} / \mathrm{s}$ and much smaller than the observed values. Currently, there is no conclusive interpretation about the large vertical winds in the observations. In this paper, strong vertical winds (above $100 \mathrm{~m} / \mathrm{s}$ ) have been simulated for the first time in GCMs. This is shown to be caused almost exclusively by an imbalance between gravity and the gradient in pressure.

[4] The model we use in this study is GITM, which is a 3-dimensional spherical code that models the Earth's thermosphere and ionosphere system using a stretched grid in latitude and altitude [Ridley et al., 2006]. There are many differences between GITM and other GCMs, and the most relevant difference for this study is that GITM solves 

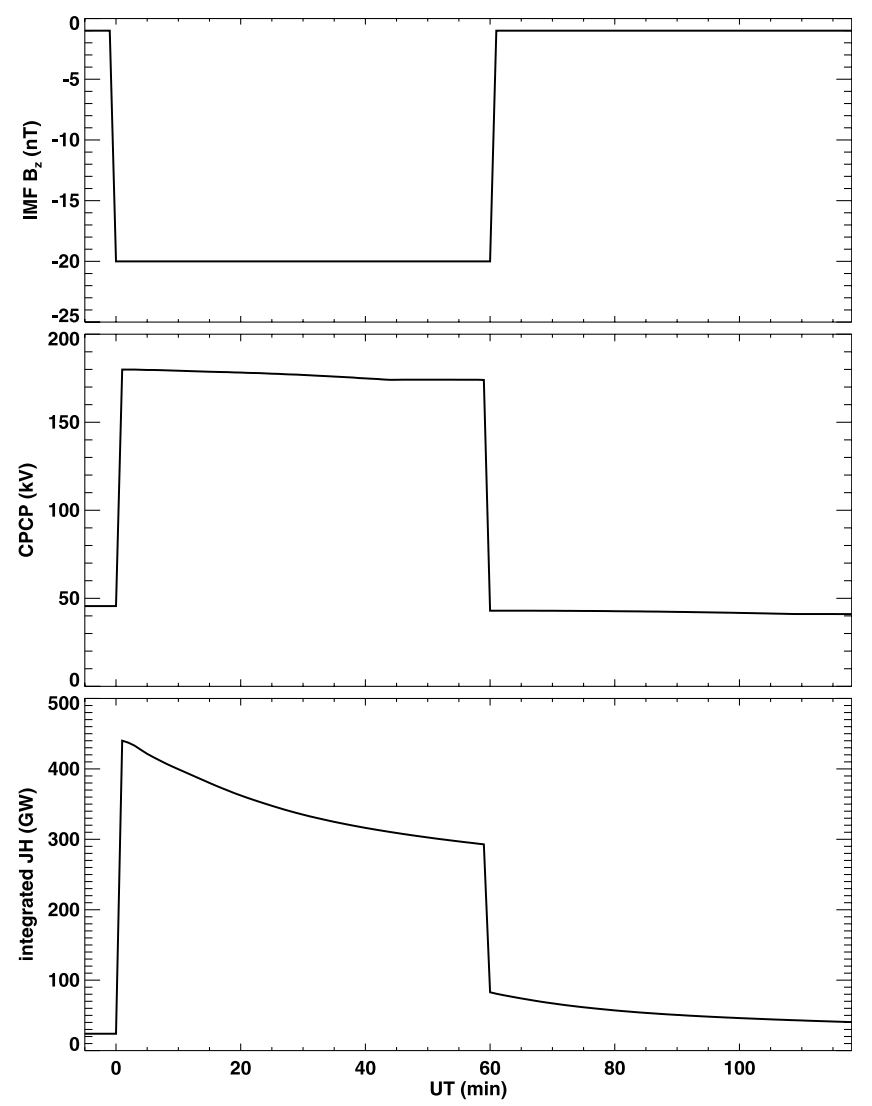

Figure 1. Temporal variation of (top) IMF $B_{z}$, (middle) CPCP, and (bottom) hemispheric integrated Joule heating in the southern hemisphere.

equation 2 instead of equation 1, as other GCMs solve. The spatial resolution for this study is $5^{\circ}$ longitude by $5^{\circ}$ latitude by $1 / 3$ scale height. This low horizontal resolution does not affect too much of the transient hydrostatic phenomena discussed in this paper, however it can potentially cause some difference for the long-period acoustic wave simulation. The temporal resolution is 2 seconds.

\section{Results}

[5] GITM has been run for 30 hours of simulation-time, reaching a quasi-steady state with quiet geomagnetic conditions (interplanetary magnetic field (IMF) $B_{z}=-1 \mathrm{nT}$, Hemispheric Power $(\mathrm{HP})=3 \mathrm{GW}$ and F10.7 $=100 \times 10^{-22}$ $\mathrm{w} / \mathrm{m}^{2} / \mathrm{Hz}$ ). The IMF $B_{z}$ is then changed to $-20 \mathrm{nT}$ with the other input parameters remaining the same and 00 UT in Figure 1 represents the time when $B_{z}$ drops to $-20 \mathrm{nT}$. The cross polar cap potential (CPCP) correspondingly increases from $45 \mathrm{kv}$ to $158 \mathrm{kv}$ in the southern hemisphere, as shown in Figure 1. Due to the correlation between IMF $B_{z}$ and the energy input from the magnetosphere to the ionosphere [Deng and Ridley, 2007], the hemispheric integrated Joule heating increases abruptly by 19 times and creates a significant disturbance in the thermosphere. After one hour, IMF $B_{z}$ returns to the previous value $(-1 \mathrm{nT})$, driving the CPCP and Joule heating to change back to the quiet condition.
[6] Temporal variations of the buoyancy acceleration $\left(-\frac{1}{\rho} \frac{\partial P}{\partial r}+g_{r}\right)$ and the vertical wind distribution in the southern hemisphere at $300 \mathrm{~km}$ altitude are shown in Figure 2. Both buoyancy acceleration and vertical wind are minimal at time 0 , which represents the quiet time. At the 3rd minute, the buoyancy acceleration shows a large upward value at locations with enhanced Joule heating. However, at 5th and 7th minutes, the buoyancy acceleration changes to be downward and maximizes at positions that correspond with regions of large upward wind seen at the $3 \mathrm{rd}$ minute. From the 15 th to 55 th minute, the buoyancy acceleration returns to the quiet value and changes little. In general, the buoyancy acceleration occurs immediately after the energy enhancement, but is short-lived since hydrostatic equilibrium quickly reasserts itself despite continued forcing. In response to the variation of buoyancy acceleration, the vertical wind increases dramatically during the first 5 minutes and decreases after that. Since the vertical wind change is equal to the time integration of the acceleration, the maximum vertical wind (5th minute) happens just after the maximum buoyancy acceleration (3rd minute). From the 15 th to 55 th minute, the vertical wind is decelerating slowly with time. The dayside continues to have relatively large vertical winds compared with the quiet time ( 0 minute), even though the buoyancy acceleration is almost zero.

[7] In order to investigate the vertical propagation of the perturbation, Figure 3 shows the temporal variations of the altitude profiles of the buoyancy acceleration and vertical wind at a specific position $\left(77.5^{\circ} \mathrm{S}, 22.5^{\circ} \mathrm{E}\right)$ in geographic coordinates, which is close to $6 \mathrm{LT}$ and shown in Figure 2a. This particular location has been chosen is because it is in the region with the maximum Joule heating [Deng and Ridley, 2007] and buoyancy acceleration in the horizontal distribution, and the longest period of large upward neutral wind, as shown in Figure 2b. Figure 3a shows that during the first minute the buoyancy acceleration increases at all altitudes. Then, there is a clear positive disturbance propagating from low altitudes to high altitudes during the 1st6.5 th minutes with the propagation speed of $1000 \mathrm{~m} / \mathrm{s}$. The reason that the disturbance propagates upward from below $150 \mathrm{~km}$ altitude is that the peak altitude of the Joule heating is close to $120 \mathrm{~km}$ altitude [Deng and Ridley, 2007]. Due to the conservation of the perturbation energy and the exponential decrease of the mass density, the disturbance caused by the sudden enhancement of energy input is transported to high altitudes with an ever magnifying amplitude. While the maximum buoyancy acceleration at $200 \mathrm{~km}$ altitude is close to $0.6 \mathrm{~m} / \mathrm{s}^{2}$, at $430 \mathrm{~km}$ it can reach $2 \mathrm{~m} / \mathrm{s}^{2}$, which is close to $25 \%$ of gravity acceleration $\left(8.7 \mathrm{~m} / \mathrm{s}^{2}\right)$ at this altitude. The lifetime of the disturbance also shows some altitude dependence. At $150 \mathrm{~km}$, the buoyancy force is disturbed from the 1 st to the 5 th minute, while at $400 \mathrm{~km}$, it expands to the 1 st -10 th minute. Overall, the buoyancy acceleration returns back to a small value $\left(<0.2 \mathrm{~m} / \mathrm{s}^{2}\right)$ after the 10 th minute.

[8] The large value of the buoyancy disturbance accelerates the thermosphere vertically, resulting in a significant vertical neutral wind. Figure $3 \mathrm{~b}$ shows that the magnitude of vertical neutral wind at $430 \mathrm{~km}$ altitude reaches $250 \mathrm{~m} / \mathrm{s}$ at the 6th minute, which is 1.5 minutes after the buoyancy acceleration maximum. These large vertical winds are very unlikely to be seen in hydrostatic models, but actually have been observed in various observations, such as by $\mathrm{DE} 2$ 
(A) buoyancy acceleration variation $\left(\mathrm{m} / \mathrm{s}^{2}\right)$

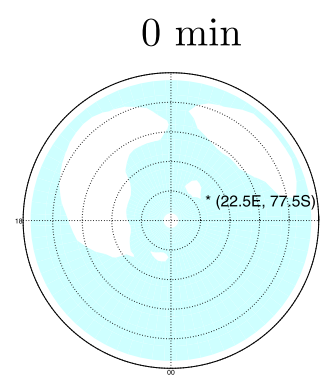

$15 \mathrm{~min}$

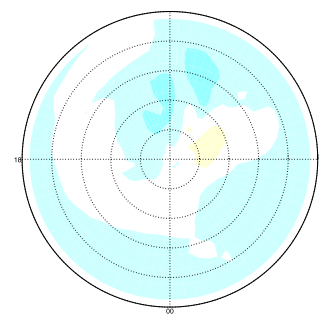

$0 \min$

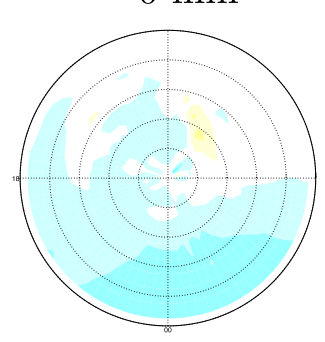

$15 \min$

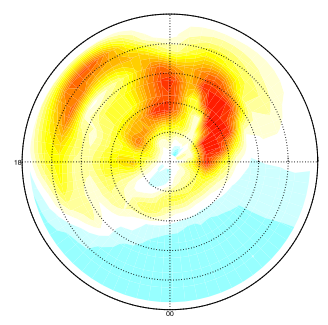

$1 \mathrm{~min}$

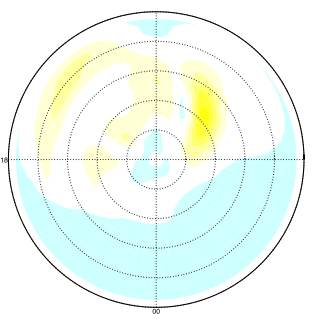

$25 \min$

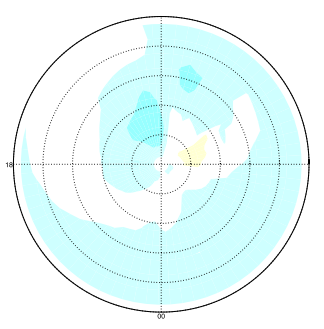

$3 \mathrm{~min}$

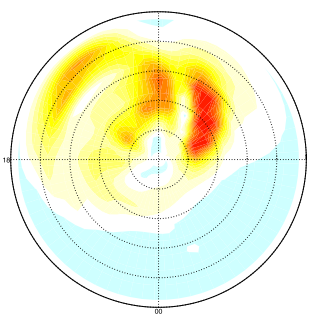

$35 \min$

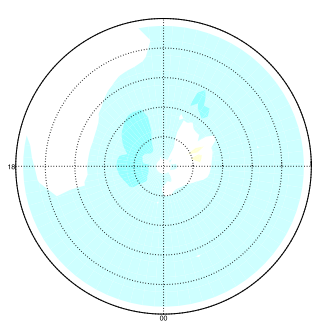

$5 \mathrm{~min}$

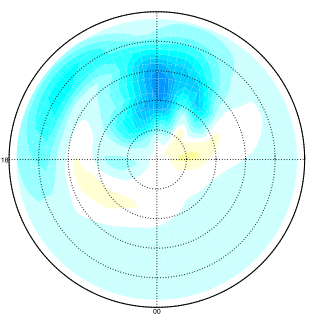

$45 \min$

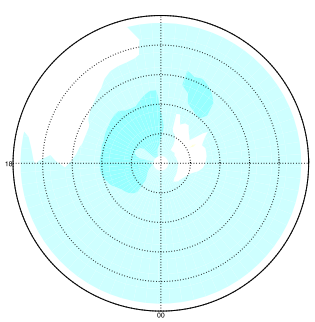

$7 \min$

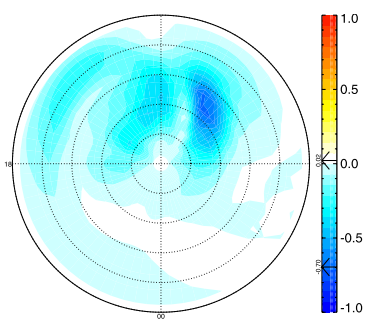

$55 \min$

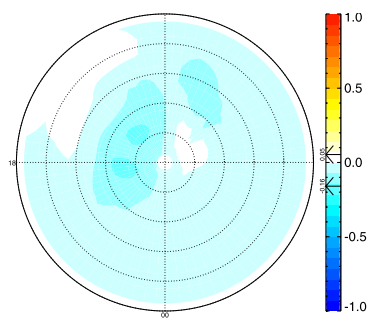

(B) Vertical wind variation $(\mathrm{m} / \mathrm{s})$

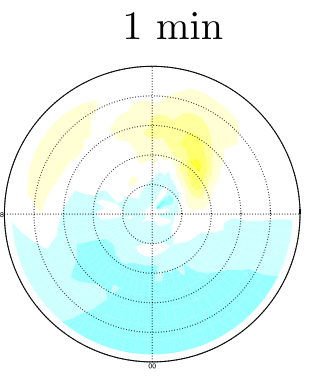

$25 \min$

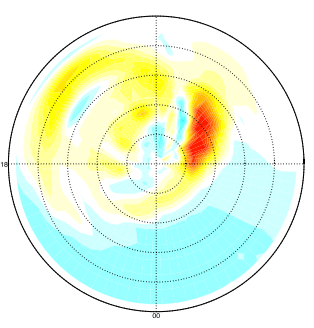

$3 \mathrm{~min}$

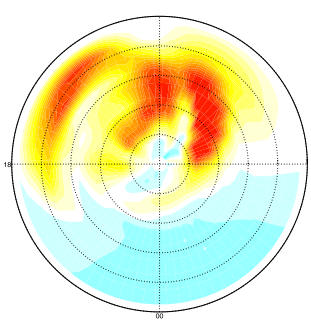

$35 \min$

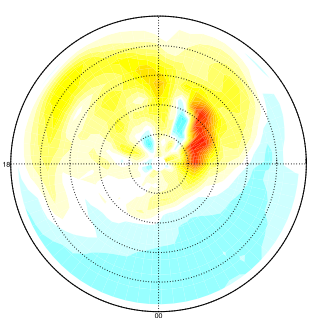

$5 \mathrm{~min}$

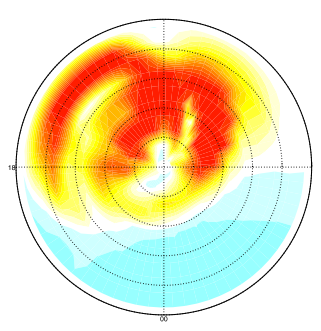

$45 \min$

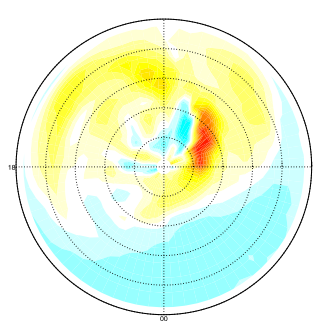

$7 \min$

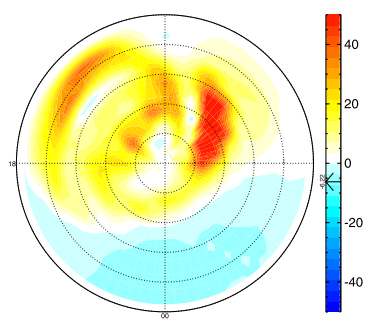

$55 \min$

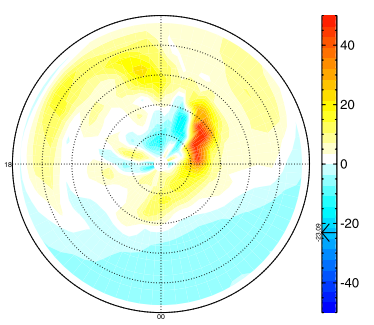

Figure 2. Temporal variation of the distributions of (a) buoyancy acceleration $\left(-\frac{1}{\rho} \frac{\partial P}{\partial r}+g_{r}\right)\left(\mathrm{m} / \mathrm{s}^{2}\right)$ and (b) vertical wind $(\mathrm{m} / \mathrm{s})$ in the southern hemisphere at $300 \mathrm{~km}$ altitude. The outside ring is $-40^{\circ}$ and the time is indicated at the top of each distribution. The left side is dusk, while the top of each plot is noon.

[Innis and Conde, 2002] and FPIs [Smith and Hernandez, 1995; Aruliah et al., 2005]. One possible reason for this large vertical wind is the non-hydrostatic effect, which is caused by the sudden intense enhancement of the energy and represented in the large value of the buoyancy acceleration $\left(2 \mathrm{~m} / \mathrm{s}^{2}\right)$. The buoyancy acceleration is negative during the 6 th-10th minutes at $430 \mathrm{~km}$, thus the upward vertical wind actually decreases with time after the 6th minute. From the 10th minute, while the buoyancy acceleration is almost zero, there is still a strong upward vertical wind with the magnitude close to $100 \mathrm{~m} / \mathrm{s}$.

[9] Due to the large vertical wind, the diffusive dissipation caused by the molecular viscosity and the thermal conduction might conceivably be significant. However, the difference between two runs with and without the vertical viscosity (not shown) in the momentum equation is quite small. This is because molecular viscosity acceleration is equal to $\eta \nabla^{2} \vec{U}$, where $\eta$ is the kinematic viscosity coefficient and $\vec{U}$ is the velocity. When the velocity is treated as a vertically propagating wave, the viscous damping effect is dependent on $\eta k_{z}^{2}=\eta \frac{4 \pi^{2}}{\lambda^{2}}$, where $k_{z}$ is the wave number and $\lambda_{z}$ is the wavelength in the vertical direction. Since the vertical wavelength of the acoustic wave is large (200 km as shown in Figure 2), $\eta \frac{4 \pi_{2}^{2}}{\lambda_{2}^{2}}$ is close to two orders of magnitude smaller than the wave frequency $(\omega=0.02 \mathrm{rad} / \mathrm{s}$ shown later) around $300 \mathrm{~km}$ altitude, and the viscous damping is not significant. Damping by thermal conduction 


\section{(A) Buoyancy acceleration $\left(\mathrm{m} / \mathrm{s}^{2}\right)$}
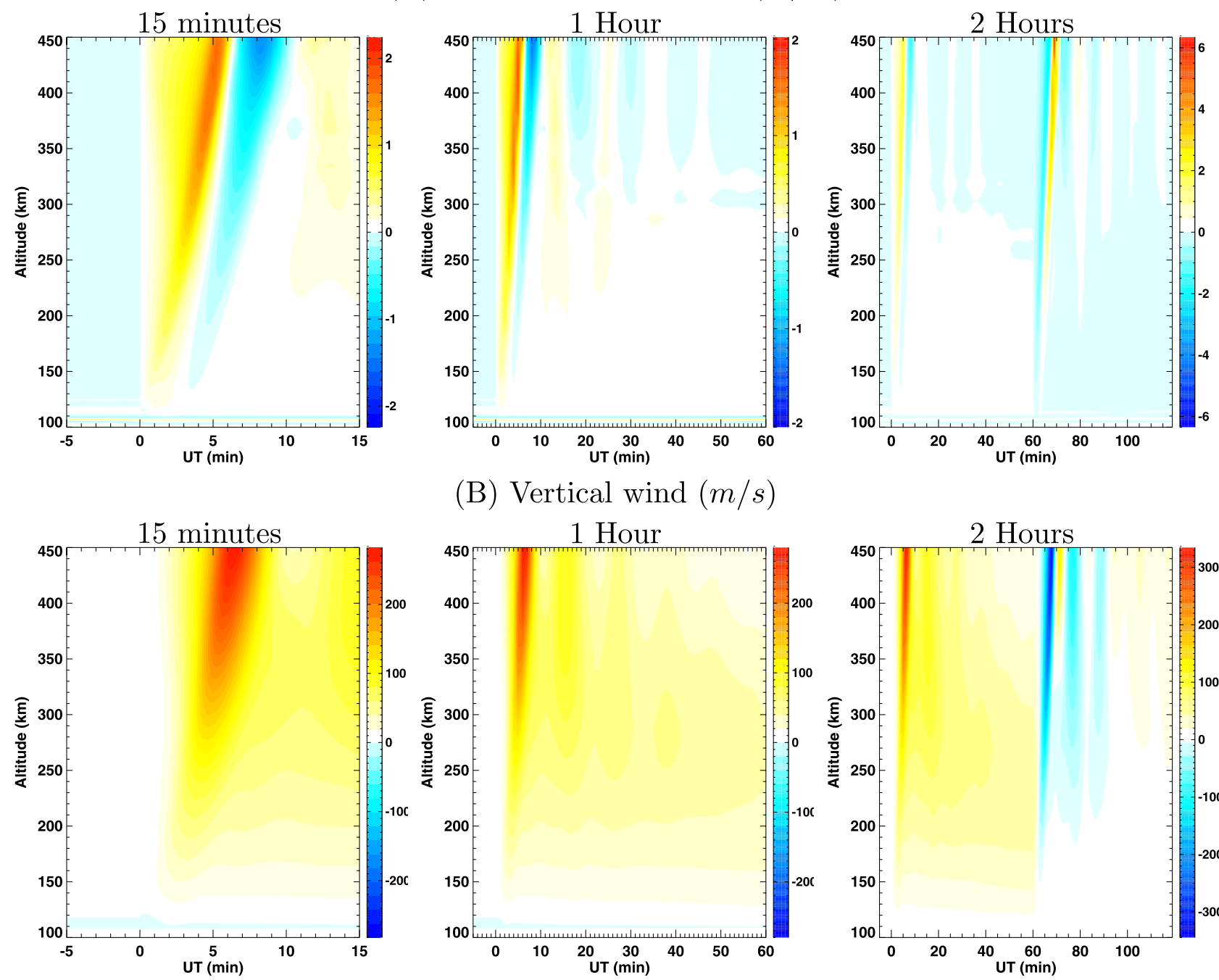

\section{(B) Vertical wind $(m / s)$}
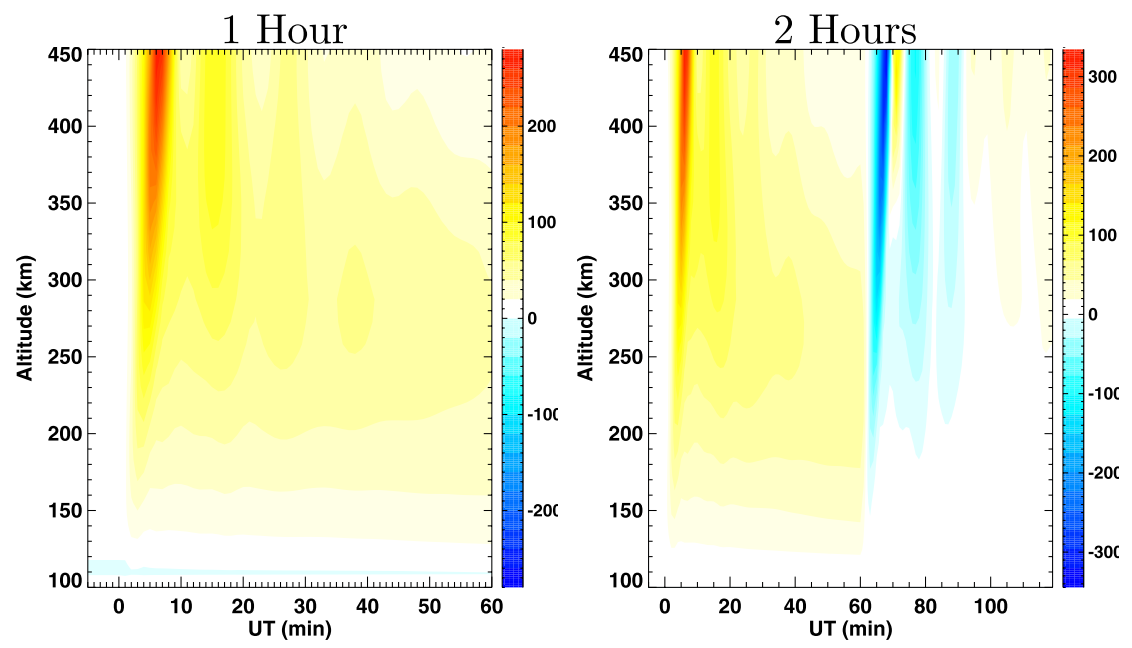

Figure 3. The time vs. altitude distribution of (a) buoyancy acceleration $\left(\mathrm{m} / \mathrm{s}^{2}\right)$ and (b) vertical wind $(\mathrm{m} / \mathrm{s})$ at $77.5^{\circ} \mathrm{S}$, $22.5^{\circ} \mathrm{E}$ during 15 minutes, 1 hour, and 2 hour time intervals. The location has been shown in Figure $2 \mathrm{a}$.

is of the same order as viscous damping [Pitteway and Hines, 1963].

[10] Figure $3 \mathrm{~b}$ (middle) shows the vertical wind decreases slowly with time during the 1 hour period when $B_{z}$ stays fixed at $-20 \mathrm{nT}$. The life time of large vertical wind $(>50 \mathrm{~m} / \mathrm{s})$ is close to the period of the enhanced forcing ( 1 hour) and much longer than that of the buoyancy acceleration disturbance $(<11$ minutes), which is caused by the variation of the pressure gradient. This difference indicates that, while the non-hydrostatic phenomena in the force term can only be sustained for a short time in the system, its impact on the vertical neutral winds lives longer and significantly influences the upper atmosphere. The upward vertical wind lifts the atmosphere and leads to a very large mass density increase at a fixed altitude. For example, at $400 \mathrm{~km}$ altitude, the density increases by a factor of two (not shown), which dramatically alters the drag on low altitude satellites. In addition, this large vertical wind introduces very significant changes in the chemical composition of both neutrals and ions at the upper heights, which has been accounted for in the GITM model.
[11] In the 1960's, some studies were done of acoustic gravity waves in the thermosphere, especially about the wave propagation from nuclear explosions [Pfeffer and Zarichny, 1962] and the ion drag effect on propagation [Hines and Hooke, 1970]. However, vertically propagating acoustic waves cannot be resolved in hydrostatic models because force balance between the pressure gradient and gravity has been assumed and used to replace the vertical momentum equation. GITM solves the complete vertical momentum equation and technically has the capability to simulate vertically propagating acoustic wave. The simulation results show that the disturbance propagation before the 10th minute is highly likely to be an acoustic wave from the phase speed, propagating direction and frequency. As calculated earlier, the phase speed is $1000 \mathrm{~m} / \mathrm{s}$, which is very close to the acoustic speed $\left(a_{s}\right)$. Both the energy and phase propagate upward, which is consistent with the dispersion relationship of acoustic waves. The frequency is calculated from the time interval between the negative and positive disturbance peaks, which occurred at times of 5.75 and 8.5 minutes at $430 \mathrm{~km}$ altitude, respectively. The difference between them represents the half period, and the frequency 
$(\omega=2 \pi / T)$ is equal to $0.02 \mathrm{rad} / \mathrm{s}$. The Brunt-Väisälä frequency $\left(N=\sqrt{(\gamma-1) \frac{g^{2}}{a_{s}^{2}}}\right)$ is close to $0.0075 \mathrm{rad} / \mathrm{s}$ at altitudes above $200 \mathrm{~km}$. Hence, $\omega \approx 3 N$, which is in the correct frequency range of an acoustic wave. When the variation of the buoyancy acceleration and the vertical wind in a one-hour time period are examined (Figure 3, middle), there are some oscillations after the 10th minute, which happen simultaneously at different altitudes and do not propagate in altitude. The oscillation frequency is close to the Brunt-Väisälä frequency, and they are more likely to be a buoyancy oscillation, instead of an acoustic wave. It is clear that after the sudden enhancement of the energy input, oscillations with different frequencies have been triggered as a ringing effect.

[12] It is also very interesting to examine the variation in a longer time period ( 2 hours), which includes both southward IMF $B_{z}$ step-up $(-1 \rightarrow-20 \mathrm{nT})$ at 0 minutes and stepdown $(-20 \rightarrow-1 \mathrm{nT})$ at 60 minutes. After $B_{z}$ returns to $-1 \mathrm{nT}$ at 60 minutes, there is a negative buoyancy acceleration disturbance followed by a positive disturbance. Subsequently, there is a large downward vertical wind during the first 15 minutes after the driver changes. Later, after the 90th minute, the vertical wind is stabilized at a small value $(<10 \mathrm{~m} / \mathrm{s})$, which is much smaller than that at the 30th minute. After the square variation of the driver (IMF $B_{z}$ ), the system returns to approximately the state in the quiet condition. During both the 30th-60th minute and the 90th-120th minute time periods, the buoyancy acceleration is very small, but the energy input levels (i.e. Joule heating) are different as shown in Figure 1 and the vertical wind magnitudes are different too. Therefore, while the buoyancy acceleration influences the temporal variation of vertical wind, the magnitude of the vertical wind in a force balanced system is related to the energy input level.

\section{Conclusion and Discussion}

[13] Under hydrostatic equilibrium, a typical assumption used in global thermosphere ionosphere models, the pressure gradient in the vertical direction is exactly balanced by the gravity force. Using the Global Ionosphere Thermosphere Model (GITM), which solves the complete vertical momentum equation, the primary characteristics of nonhydrostatic effects on the upper atmosphere are investigated. Our results show that after a sudden intense enhancement of high-latitude Joule heating, the vertical pressure gradient force can locally be $25 \%$ larger than the gravity force, resulting in a significant disturbance away from hydrostatic equilibrium. This disturbance is transported from the lower altitude source region to high altitudes through an acoustic wave, which has been simulated in a global circulation model for the first time. Due to the conservation of perturbation energy, the magnitude of the vertical wind perturbation increases with altitude and rea- ches $150(250) \mathrm{m} / \mathrm{s}$ at $300(430) \mathrm{km}$ during the disturbance. The upward neutral wind lifts the atmosphere and raises the neutral density at high altitudes by more than $100 \%$. While the time scale of the buoyancy acceleration perturbation is around 5-10 minutes in this case, the large vertical wind (above $50 \mathrm{~m} / \mathrm{s}$ ) at $300 \mathrm{~km}$ altitude lasts for a significantly longer time, and depends on the lifetime of the forcing. These large vertical winds are observed and are not typically reproduced by hydrostatic models of the thermosphere and ionosphere. The lack of non-hydrostatic effect and the corresponding large vertical winds in hydrostatic GCMs is one possible reason for the underestimate of neutral density enhancements during storm times in the simulations.

[14] Acknowledgments. This research was supported by NSF through grants ATM0639336 NASA grant NN604GKL86 and NASA Sun-Earth Connection Theory Program. H.-L. Liu's effort is in part supported by the Office of Naval Research (N00014-07-C0209). National Center for Atmospheric Research (NCAR) is supported by National Science Foundation (NSF).

\section{References}

Aruliah, A. L., et al. (2005), First direct evidence of meso-scale variability on ion-neutral dynamics using co-located tristatic FPIs and EISCAT radar in Northern Scandinavia, Ann. Geophys., 23, 147-162.

Deng, Y., and A. J. Ridley (2007), Possible reasons for underestimating Joule heating in global models: $E$ field variability, spatial resolution, and vertical velocity, J. Geophys. Res., 112, A09308, doi:10.1029/2006JA012006.

Dickinson, R. E., E. C. Ridley, and R. G. Roble (1981), A threedimensional, time-dependent general circulation model of the thermosphere, J. Geophys. Res., 86, 1499-1512.

Fuller-Rowell, T. J., and D. Rees (1980), A three-dimensional, timedependent, global model of the thermosphere, J. Atmos. Sci., 37, $2545-2567$.

Hines, C. O., and W. H. Hooke (1970), Discussion of ionization effects on the propagation of acoustic gravity waves in the ionosphere, J. Geophys. Res., 75, 2563-2568.

Innis, J. L., and M. Conde (2002), Characterization of acoustic-gravity waves in the upper thermosphere using Dynamics Explorer 2 Wind and Temperature Spectrometer (WATS) and Neutral Atmosphere Composition Spectrometer (NACS) data, J. Geophys. Res., 107(A12), 1418, doi:10.1029/2002JA009370.

Pfeffer, R. L., and J. Zarichny (1962), Acoustic gravity wave propagation from nuclear explosions in the Earth's atmosphere, J. Atmos. Sci., 19, 256-263.

Pitteway, M. L. V., and C. O. Hines (1963), The viscous damping of atmospheric gravity waves, Can. J. Phys., 41, 1935-1948.

Price, G. D., R. W. Smith, and G. Hernandez (1995), Simultaneous measurements of large vertical winds in the upper and lower thermosphere, J. Atmos. Terr. Phys., 57, 631-643.

Ridley, A. J., Y. Deng, and G. Tóth (2006), The global ionospherethermosphere model, J. Atmos. Sol. Terr. Phys., 68, 839-864.

Skamarock, W. C., and J. B. Klemp (2007), A time-split nonhydrostatic atmospheric model for research and NWP applications, J. Comput. Phys., in press.

Smith, R. W., and G. Hernandez (1995), Vertical winds in the thermosphere within the polar cap, J. Atmos. Terr. Phys., 57, 611-620.

Y. Deng, H.-L. Liu, and A. D. Richmond, High Altitude Observatory, National Center for Atmospheric Research, P.O. Box 3000, Boulder, CO 80307-3000,USA. (ydeng@ucar.edu; liuh@ucar.edu; richmond@ucar.edu)

A. J. Ridley, Center for Space Environment Modeling, University of Michigan, 1416 Space Research Building, 2455 Hayward Street, Ann Arbor, MI 48109-2143, USA. (ridley@umich.edu) 